\title{
The CMS Calorimeter in 2007: Performance and Physics Goals
}

\author{
Paul R. Lecoq, Member, IEEE
}

\begin{abstract}
Thirteen years after the decision to build a Lead Tungstate based electromagnetic calorimeter for the CMS experiment at the Large Hadron Collider (LHC) at CERN the detector is now in its final assembly and installation phase for the LHC startup in early 2008. It is made of 75848 Lead Tungstate crystals, 61200 of 133 cubic centimeter for the barrel and 14648 of 189 cubic centimeter for the endcaps, for a total weight of 90 tons of crystals. This challenging project aims to achieve an extreme precision in photons and electrons energy measurement. General motivations, main technical challenges, measured performances, and the actual status of the project will be discussed.
\end{abstract}

Index Terms-Calorimeter, crystal, energy resolution, scintillator.

\section{INTRODUCTION}

$\mathbf{T}$ HE Compact Muon Solenoid (CMS) detector is one of the two general purpose detectors being installed at the Large Hadron Collider (LHC) at CERN. The installation in the underground cavern is now well advanced and should be completed by the end of this year for a first physics run in 2008. It is built around a 4 Tesla superconducting magnet surrounding a high spatial resolution Silicon Tracker close to the collider interaction point for charge particle tracking and momentum measurement, followed by a homogeneous crystal based electromagnetic calorimeter (ECAL) for very precise energy measurement of gammas and electrons and by a hadron calorimeter made of a sandwich of brass and plastic scintillator plates to allow the measurement of hadron jets. Outside of the coil the return yoke of the magnet is fully instrumented with a set of high resolution drift chambers for a precise evaluation of the momentum of muons. The CMS detector has an overall diameter of 15 meters, a length of 22 meters for a total weight of about 12,500 tons.

The purpose is to measure as precisely as possible the decay products of unstable particles produced by the LHC $14 \mathrm{TeV}$ proton-proton interactions. The high luminosity and high energy of the LHC give access to rare physics processes. Among them is the search for the origin of mass in the universe through the discovery of the Higgs boson as predicted by the Standard Model (SM). Another exciting perspective is the quest for supersymmetric particles, as a signature of a high level symmetry between the world of fermions and the world of bosons, which could open interesting perspectives in the understanding of the dark matter puzzle.

Manuscript received June 30, 2007; revised October 9, 2007. This contribution is made on behalf of the CMS-ECAL group.

The author is with CERN, CH-1211 Geneva 23, Switzerland (e-mail: paul. lecoq@cern.ch).

Color versions of one or more of the figures in this paper are available online at http://ieeexplore.ieee.org.

Digital Object Identifier 10.1109/TNS.2007.910973

\section{Physics GoAls AND REQUIRED PERFORMANCE FOR THE ELECTROMAGNETIC CALORIMETER}

Indications from the LEP experiments seem to favor a relatively low mass for the SM Higgs boson, typically in a mass range between 120 and $150 \mathrm{GeV} / \mathrm{c}^{2}$. In this mass range the Higgs can be best detected through its $\gamma \gamma$ decay channel. The reaction $\mathrm{H} \rightarrow \gamma \gamma$ has therefore been used as a benchmark for the design of the ECAL.

This is indeed a very challenging reaction as the branching ratio for this decay channel is very low. Moreover the natural width of such a low mass Higgs is small and the signal is contaminated by an irreducible background made of a large number of $\gamma$ rays produced by different physics reactions. The discovery potential of such a particle will therefore be essentially driven by the experimental energy and mass resolution of the calorimeter. One important aspect of it is a high granularity to resolve two close by $\gamma$ rays produced by the decay of abundant $\pi^{\circ}$.

To add to the complexity the LHC works at a high frequency of $40 \mathrm{MHz}$, necessitating a time to form and collect the signals of not more than $25 \mathrm{~ns}$ to avoid overlaps. The high luminosity of the machine produces high radiation fluence, which will amount to $10^{13} \mathrm{n} / \mathrm{cm}^{2}$ and $1 \mathrm{kGy}$ in the central region and to $2 \times 10^{14} \mathrm{n} / \mathrm{cm}^{2}$ and $50 \mathrm{kGy}$ in the forward region for 10 years of operation. A good radiation tolerance of the detector is mandatory to maintain its stability in such an environment. This concerns the active calorimeter material but also the electronics, a large part of it being integrated in the detector itself. Finally the whole detector is immersed in a $4 \mathrm{~T}$ magnetic field, which imposes severe constraints on some of the readout components.

\section{TECHNICAL CHOICES AND PROGRESS IN THE CONSTRUCTION}

The combined requirements for an ultimate energy resolution of $0.5 \%$ at high energy and for a high granularity has led to the choice of a homogeneous calorimeter with Lead Tungstate crystals $\left(\mathrm{PbWO}_{4}\right.$ or in short: $\left.\mathrm{PWO}\right)$. The CMS electromagnetic calorimeter [1] comprises a barrel made of $61200 \mathrm{PWO}$ crystals, with a tapered shape and mean dimensions of $2.3 \times 2.3 \times 23 \mathrm{~cm}^{3}$ and two endcaps of 7324 crystals each, also tapered with dimensions of about $3.0 \times 3.0 \times 22 \mathrm{~cm}^{3}$. In front of the endcap crystals a preshower detector made of a Lead/Silicon sandwich helps to identify $\pi^{\circ}$ through their decay into two close by gamma photons. Fig. 1 shows the general layout of the ECAL. To avoid cracks aligned with particle trajectories, the axes of the crystals are tilted by $3^{\circ}$ both in $\mathrm{r}-\mathrm{z}$ plane and in azimuthal direction with respect to the direction from the interaction point. The crystal length corresponds to 25.8 radiation lengths in the barrel. The crystals are grouped in subunits of $5 \times 2$, held together by a glass fiber alveolar structure. Submodules are mounted in 


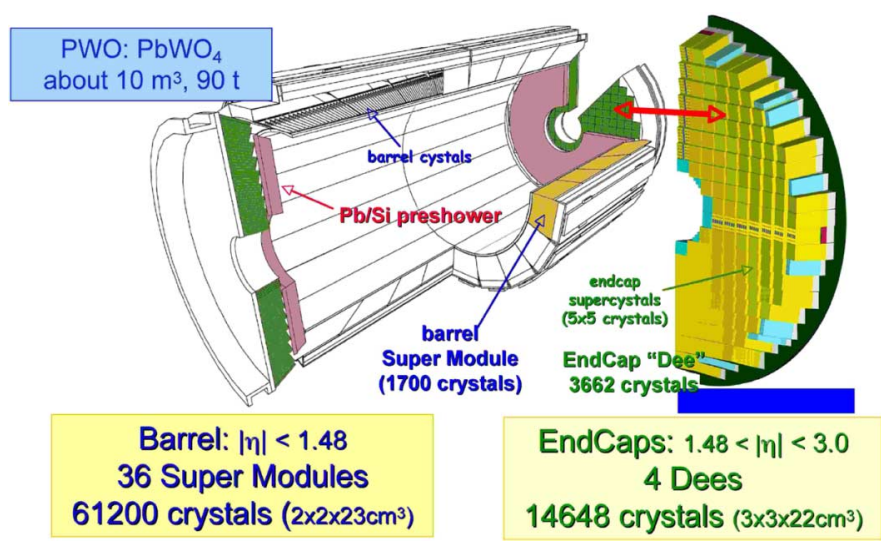

Fig. 1. Layout of the CMS electromagnetic calorimeter.

groups (modules) of 40 or 50 on an aluminum element (grid), which holds their weight from the back. Four modules are assembled together in a supermodule, comprising 1700 crystals. Eighteen supermodules form each of the two half barrels. A similar construction scheme is applied for the endcaps, where crystals are organized in $5 \times 5$ subunits in a carbon fiber alveolar structure.

\section{A. Crystals}

The choice of Lead Tungstate was motivated by its high density $\left(8.28 \mathrm{~g} / \mathrm{cm}^{3}\right)$. It has indeed a short radiation length $(0.89 \mathrm{~cm})$ and a small Moliere radius $(2 \mathrm{~cm})$ allowing a compact design with a high granularity. Moreover its broad light emission band peaking in the visible at $420 \mathrm{~nm}$ is adequate for the majority of the photodetectors and its short decay time of $17 \mathrm{~ns}$ allows an almost full collection of the light in $100 \mathrm{~ns}$. However this crystal is working in a strong temperature quenching mode at room temperature, which strongly reduces its intrinsic light emission to about 50 photons $/ \mathrm{MeV}$. The consequence is that photodetectors with internal gain are required, which can operate in the strong magnetic field of the experiment.

Furthermore, the PWO light yield decreases by $2 \%$ when the temperature increases by $1^{\circ} \mathrm{C}$ at room temperature. To maintain the energy resolution at the specification value $(0.5 \%$ at high energy) a large engineering effort had to be deployed for the design of a cooling system allowing to maintain the whole 90 tons calorimeter within $0.1^{\circ} \mathrm{C}$ around the working temperature of $18^{\circ} \mathrm{C}$ [2].

Over the past 15 years considerable efforts have been deployed to understand the properties and to optimize the performance of PWO for the tight CMS specifications. The conditions to grow large and color-free crystals, to suppress slow components and to reach unprecedented radiation tolerance levels, have been particularly investigated. In addition the development of mass production technologies (crystal growth and mechanical processing) allowing this huge production to be made with the highest production yield (and therefore a controlled cost) has been considered with a high priority. A review of this work and on PWO properties is given in [3].

About $95 \%$ of the crystals are produced in Russia (Bogoroditsk Techno-Chemical Plant) using the Czochralsky

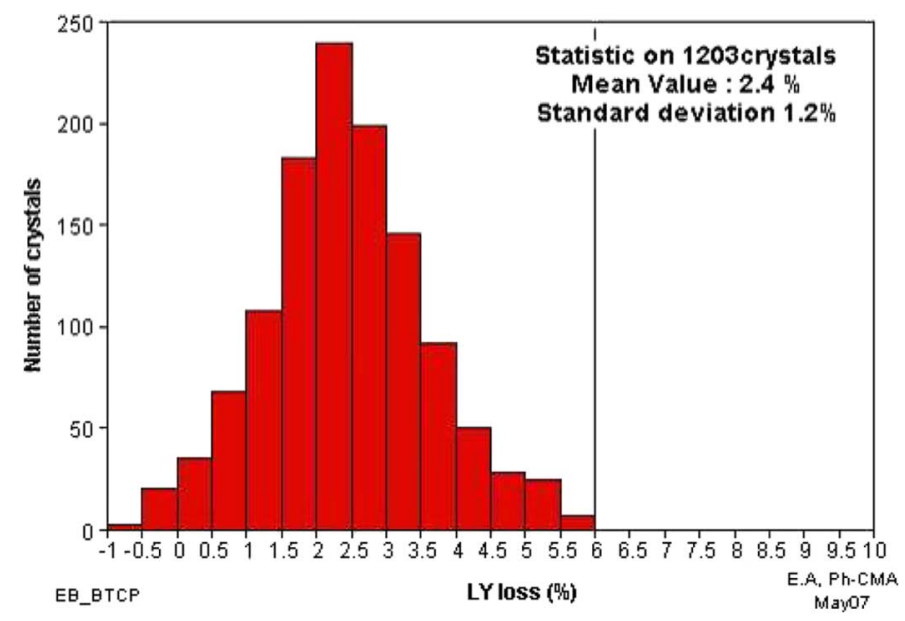

Fig. 2. Light yield loss for 1203 barrel crystals randomly chosen and front irradiated with a ${ }^{60} \mathrm{Co}$ source at a dose rate of $0.15 \mathrm{~Gy} / \mathrm{h}$.

technology, the remaining crystals being produced in China (Shanghai Institute of Ceramics) by the Bridgeman method.

The barrel production is complete since February 2007 with an impressive production rate in the last year of about 1250 crystals per month. The quality is excellent with most of the performance well above the specification value, as a result of a strict quality control procedure [4]. This is illustrated on Fig. 2 for the low dose rate radiation damage behavior of 1203 crystals randomly chosen, showing an average light yield loss of $2.4 \%$, well below the $6 \%$ set in the specifications. The endcap crystal production in now well on the way and will be completed in March 2008.

\section{B. Photodetectors}

The low light yield of PWO requires the use of photodetectors with an internal gain, which can operate in the 4 Tesla field of the experiment. The radiation level in the barrel allows the use of silicon based avalanche photodiodes (APD) [5]. Two $5 \times 5 \mathrm{~mm}^{2}$ APDs produced by Hamamatsu are mounted at the rear of each barrel crystal in a white plastic capsule. They operate at a gain of 50, where their excess noise factor is maintained at a value of about 2. At the PWO wavelength emission their quantum efficiency is about $75 \%$. However their temperature dependence of $-2.4 \% /{ }^{\circ} \mathrm{C}$ (in the same direction as the crystals) imposes stringent requirements on the temperature stabilization of the detector. Furthermore a high gain dependence on bias voltage $(3 \% / \mathrm{V})$ requires a high stability of the high voltage supplies.

Presently all the APDs $(122,400)$ have been delivered, fully characterized and glued on the crystals.

For the endcaps the radiation levels are too high to safely operate Silicon based detectors like APDs and vacuum phototriodes (VPTs) have been chosen instead [6]. The choice of short vacuum tubes is indeed possible in this region as the magnetic field is almost aligned with the tube electrostatic field. The VPTs have all been produced by Research Institute Electron in St Petersburg and individually characterized. They have a diameter of $26.5 \mathrm{~mm}$ and operate with a gain of about 10 with a quantum efficiency of about $20 \%$. In the 4 Tesla magnetic field the gain is reduced by only $10 \%$. 


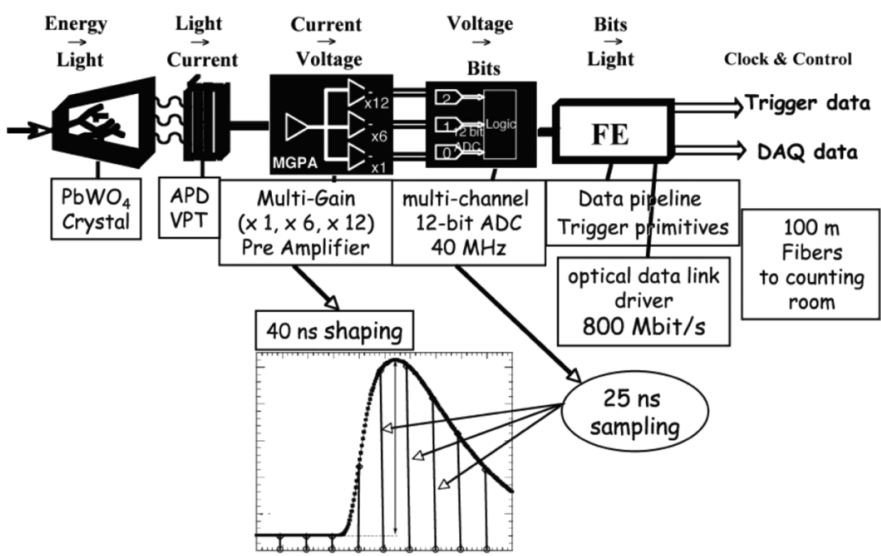

Fig. 3. ECAL readout and trigger architecture.

\section{Read-Out Electronics}

The readout and trigger electronics of the ECAL is divided into two parts: the on-detector electronics located inside the CMS detector and the off-detector electronics located outside the detector in the underground service cavern [7].

The on-detector part is highly integrated with the cooling system just in the back of the crystals. It is designed in IBM CMOS $0.25 \mu \mathrm{m}$ technology in order to achieve the required radiation tolerance. The photodetector signal is first going through a low noise, $40 \mathrm{~ns}$ shaping preamplifier. It is then split in 3 parallel lines through amplifiers with gains 1, 6 and 12 and is digitized at a sampling frequency of $40 \mathrm{MHz}$ in 12-bit ADCs to provide a dynamic gain of 40,000. The digitized data as well as energy sums generated at this level are then stored in pipelines in groups of 5 crystals during the level 1 trigger latency. Both DAQ and trigger data are then transmitted to the off-detector electronics in the counting room through 9000 high-speed $(800 \mathrm{Mb} / \mathrm{s})$ serial links over a distance of $100 \mathrm{~m}$. These links and the corresponding opto-electronics must be resistant to the stringent radiation conditions of the detector area.

The off-detector electronics of the CMS electromagnetic calorimeter is designed to provide to the trigger system the required information for level 1 accept decision in the short time budget and to reduce the event size by a factor of about 20. For each supermodule there is a Trigger Concentrator Card (TTC) and a Data Concentrator Card (DCC) both under the control of a Clock and Control System card.

The layout of the ECAL electronics is shown in Fig. 3.

All the electronics of the barrel has been delivered and installed and is presently in the commissioning phase. Some parts of the endcap trigger system are still to be produced.

\section{Laser Monitoring System}

The crystals will be exposed to high radiation levels during the LHC operation. In spite of their good radiation hardness their optical transparency is expected to drop by a few percents as a result of the formation of color centers. However the scintillation mechanism is not affected by the radiation. It is therefore sufficient to track and monitor the transmission loss with a laser monitoring system. For this purpose a fiber distributed laser

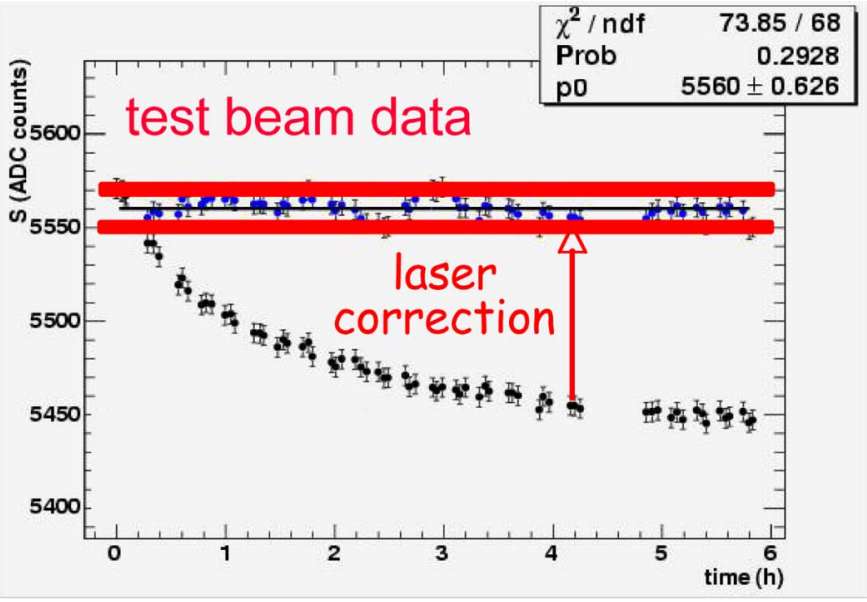

Fig. 4. Effect of the laser monitoring correction on test beam data.

system has been developed, injecting calibrated light pulses in each crystal at a frequency of $80 \mathrm{~Hz}$ during data taking, exploiting the $3 \mu \mathrm{s}$ gap during the LHC bunches. The system operates at $440 \mathrm{~nm}$ (close to the PWO peak emission wavelength) and at $796 \mathrm{~nm}$, where no damage is expected in order to correct for possible systematic effects. It is now fully installed and operational on the barrel and test beam results, where crystals have been purposely irradiated, show that it perfectly fulfills the requirements of a few per mil stability (Fig. 4) [8].

\section{CAlorimeter Performance}

Since several years more and more complete parts of the calorimeter have been regularly exposed in test beams to understand its behavior and evaluate its performance. Recently fully equipped supermodules have been calibrated with electrons of different energy [9].

The achieved energy resolution for a $3 \times 3$ crystal matrix with the beam aiming in the central area of the central crystal can be parametrized by the following formula:

$$
\frac{\sigma(E)}{E}=\frac{2.8 \%}{\sqrt{E}} \oplus \frac{0.125}{E} \oplus 0.3 \%
$$

The stochastic term of $2.8 \%$ is mainly driven by the lateral containment fluctuation of the showers in a $3 \times 3$ crystals matrix as well as by the photostatistics in the scintillator and photodetector.

The noise term, which determines the behavior in the low and medium energy range, is excellent. A single channel noise of $40 \mathrm{MeV}$ has been measured and the $125 \mathrm{MeV}$ reported here for a $3 \times 3$ matrix shows that there is no correlated noise between the channels.

The constant term determines the high energy resolution of the calorimeter. It is the result of a number of systematic effects in the calorimeter, among which the main contributors are:

- energy fluctuations due to incomplete shower containment: front and rear leakage, dead material between crystals,

— light yield uniformity effects related to the longitudinal shower statistical fluctuations in the crystal 


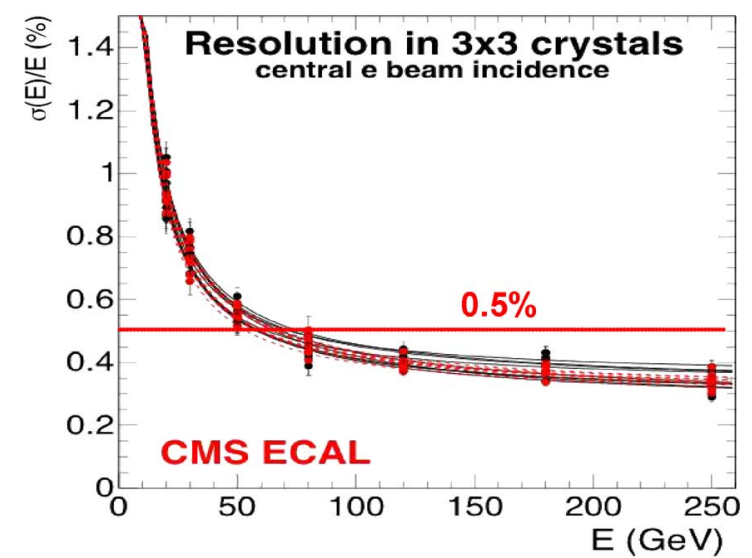

Fig. 5. Energy resolution curves for several crystals of completed supermodules as measured in a test beam prior to installation.

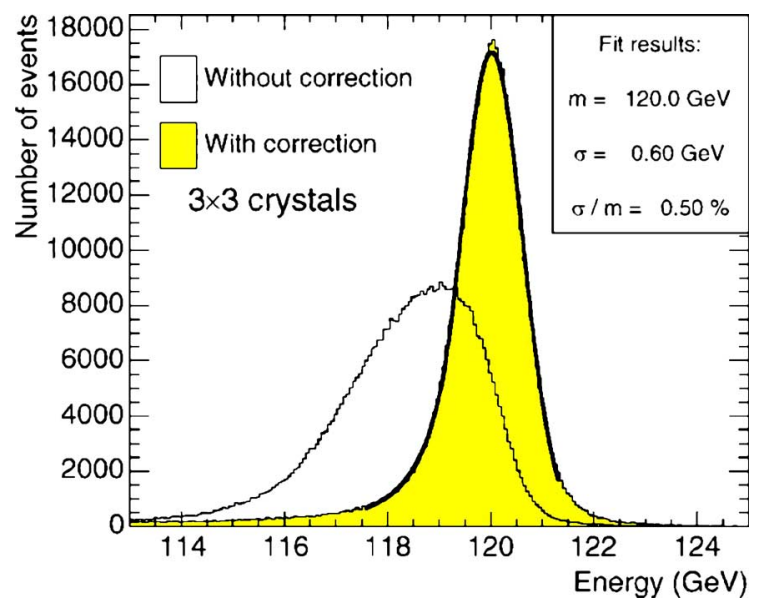

Fig. 6. $120 \mathrm{GeV}$ electron energy distribution curve before and after correction for the inter-crystal cracks.

— effect of residual temperature fluctuations on crystal light response and APD gain

- effect of residual high voltage fluctuations on APD gain

- intercalibration errors

A great deal of engineering efforts have been spent to control these systematic effects at the minimum possible level. As a result of this, an excellent value of $0.3 \%$ has been achieved for the constant term on individual $3 \times 3$ crystal matrices (i.e. without intercalibration errors).

An important aspect of the performance of the detector is the reproducibility of the results for all the crystals of the calorimeter. Fig. 5 shows that the excellent energy resolution curve is indeed nicely reproduced for several crystals randomly chosen.

It remained to be seen that this excellent energy resolution could be reproduced for any impact point on the front face of the crystals. Due to unavoidable cracks between crystals, although reduced to the strict minimum by a proper mechanical design, the energy response for a $3 \times 3$ crystal matrix drops by nearly $2 \%$ when the impact point approaches the crack. As the calorimeter is designed to measure not only electrons but also gamma photons we cannot rely on tracker information and specific algorithms have been developed to infer the impact point of

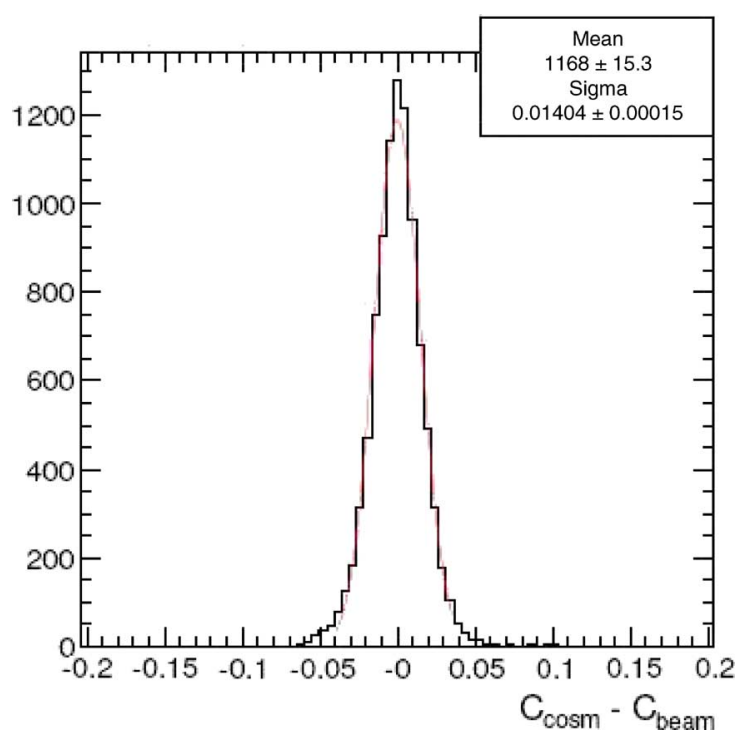

Fig. 7. Distribution of the difference between cosmic and test beam intercalibration constants.

these $\gamma$ photons on the front face of the crystals from the energy distribution on the different crystals [10]. Fig. 6 shows the energy distribution curve for $120 \mathrm{GeV}$ electrons on a crystal where no cut has been applied on the impact point. The distribution before and after correction are shown, illustrating that the design figure of $0.5 \%$ energy resolution is actually achieved for any incoming high energy electron or photon in the crystals.

\section{STATUS OF CONSTRUCTION AND COMMISSIONING}

The discovery potential of the ECAL depends critically on a good control of the intercalibration errors. The ECAL community has developed a several step strategy, as part of the commissioning process, to approach as quickly as possible in the early LHC running phase the required value of better than $0.5 \%$ for inter-channel calibration.

The first step is based on a precise evaluation during the construction of all the parameters affecting the calorimeter response. The main driver is the crystal light yield with a variation of up to $30 \%$ for all the crystals produced. The APD and electronics gain spread has also been considered but its contribution is small. The precision of the systematic laboratory checks on all the critical components during the construction has allowed to reduce the dispersion of the calibration constants to $4.5 \%$.

Due to the lack of test beam time only 10 of the 36 supermodules have been calibrated in an electron test beam with an intercalibration defined at the level of better than $0.5 \%$.

To obtain a better evaluation of the intercalibration coefficients than from construction data each of the 36 supermodules has been installed at the end of their assembly on a cosmic bench and the response of each crystal has been measured with minimum ionizing cosmic muons with proper selection for full path-length in the crystal. The dispersion of the corrected crystal response can be reduced to $1.5 \%$ with this method as compared to test beam calibration (Fig. 7). 


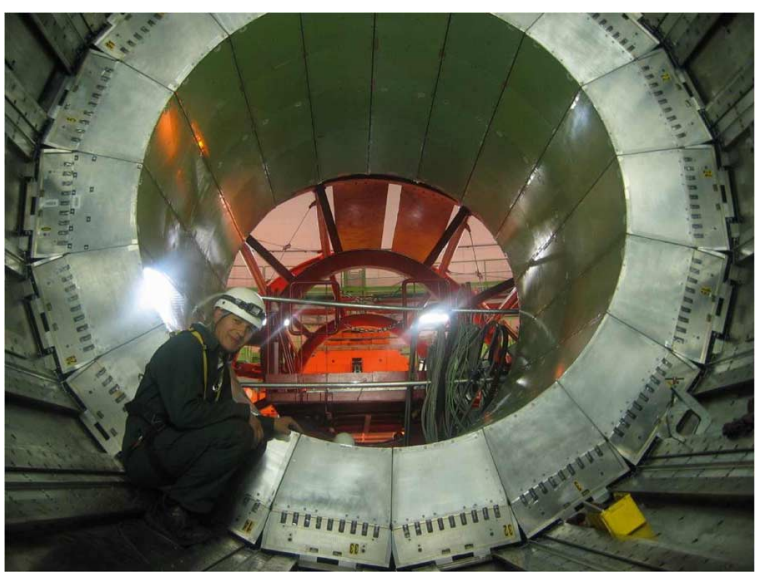

Fig. 8. ECAL 1st half barrel installed in the CMS hadron calorimeter.

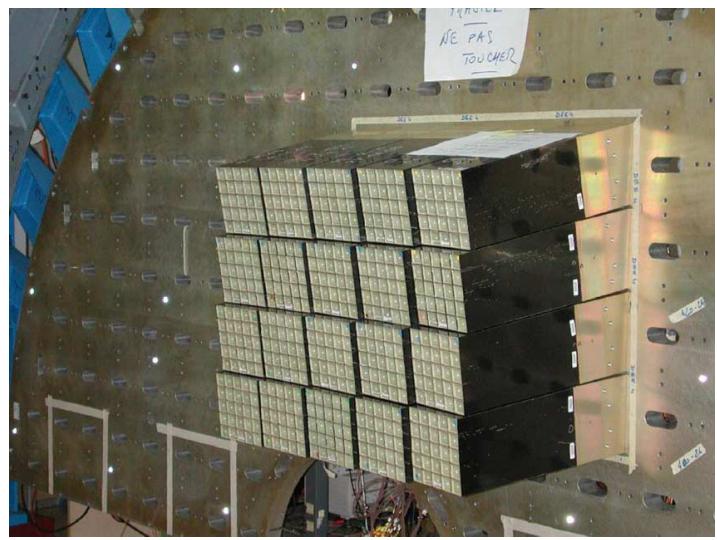

Fig. 9. First 20 endcap supercrystals mounted on their Dee plate.

All this effort is motivated by the need to start LHC operation with an estimate as good as possible of the intercalibration constants, even if a number of well known physics processes will allow a continuous tuning of these parameters during physics runs. Among them the electrons from the decay of the $\mathrm{W}$ boson will be used for a cross-check of the reconstructed energy from the ECAL with the momentum measured by the tracker. Narrow width resonances such as the $\pi^{\circ}$ or $\eta$ decay into two $\gamma$ will also be used for this purpose, allowing an intercalibration independent from the tracker.

Today the barrel construction is completed and the first half barrel is already installed in the experiment. Fig. 8 shows the 18 first supermodules in their final position inside the hadron calorimeter. The second half will be installed in July 2007.
The endcap construction is now well on the way with an installation foreseen in the first half of 2008. Fig. 9 shows the first 20 supercrystals ( 25 crystals each) mounted on the first Dee plate.

\section{CONCLUSION}

After nearly 15 years of intense R\&D, production and tests of a huge quantity of several components at the cutting edge of technology the CMS electromagnetic calorimeter is now in the final phase of its construction. All of the barrel is built and the first half of it installed in the experiment. The construction of the endcaps is well on the way and installation is scheduled to start in the next few months.

\section{ACKNOWLEDGMENT}

This presentation has been made on behalf of the CMS ECAL collaboration. The author is grateful to all his colleagues from this CMS ECAL community. He also would like to pay a special tribute to the Crystal Clear collaboration and more generally to the whole SCINT community, whose contributions, direct or indirect, have been essential in the development of the Lead Tungstate crystal at the excellent level of quality consistently achieved today.

\section{REFERENCES}

[1] CMS Collaboration, The Electromagnetic Calorimeter Technical Design Rep. CERN/LHCC 97-033 (1997). CMS TDR 4, Addendum CERN/LHCC 2002-027, 2002.

[2] O. Teller et al., "The design of the cooling system for the CMS barrel electromagnetic calorimeter," in Proc. Nucl. Sci. Symp. Conf. Rec., Oct. 2006 , vol. 1 , pp. $430-434$

[3] A. Annenkov et al., "Lead tungstate scintillation material," Nucl. Instrum. Meth. Phys. Res. A, vol. 490, pp. 30-50, 2002.

[4] E. Auffray et al., "Overview of the 63000 PWO barrel crystals for CMS_ECAL production," IEEE Trans. Nucl. Sc., contribution to this conference.

[5] K. Deiters et al., "Radiation hard avalanche photodiodes for CMS ECAL," Nucl. Instrum. Meth. Phys. Res. A, vol. 504, pp. 44-47, 2003.

[6] K. W. Bell et al., "The development of vacuum phototriodes for the CMS electromagnetic calorimeter," Nucl. Instrum. Meth. Phys. Res. A., vol. 469, pp. 29-46, 2001.

[7] R. Alemany et al., "Overview of the ECAL of-detector electronics of the CMS experiment," IEEE Trans. Nucl. Sci., vol. 52, no. 5, pp. 1918-1924, Oct. 2005.

[8] L. Zhang et al., "Performance of the light monitoring system of the monitoring light source for the CMS lead tungstate crystal calorimeter," IEEE Trans. Nucl. Sci., vol. 52, no. 4, pp. 1123-1130, Aug. 2005.

[9] P. Adzic et al., CMS Electromagnetic group, "Results of the first performance tests of the CMS electromagnetic calorimeter," Eur. Phys. J. C, vol. 44, pp. 1-25, 2006, s02.

[10] CMS Electromagnetic groupP. Adzic et al., "Energy resolution of the barrel of the CMS electromagnetic calorimeter," Institute of Physics Publishing and SISSA 2007 JINST 2 P04004. 